\title{
A NEW GRAVIMETRIC DETERMINATION OF THE GEOID OF THE BRITISH ISLES
}

\author{
W. E. Featherstone \\ Curtin University of Technology, Perth, Western Australia
}

\author{
J. G. Olliver
}

Oxford University

\begin{abstract}
A new gravimetric detcrmination of the geoid of the British Isles has been made, using a modified fersion of Stokes integral in combination with a global geopotential model and a digital terrain model
\end{abstract}

\section{INTRODUCTION}

The Global Positioning System determines positions very accurately in a terrestrial three-dimensional Cartesian frame, and the resulting $X, Y, Z \mathrm{co}$ ordinates are easily convertible into ellipsoidal co-ordinates - latitude, longitude and height above the ellipsoid [7]. However, the conversion of ellipsoidal height into a meaningful physical quantity, namely orthometric height or height above the geoid requires a correspondingly accurate knowledge of the geoid-ellipsoid separation, or geoid height, as given by the equation

$$
H=h-N
$$

where $H$ is orthometric height, $h$ ellipsoidal height and $N$ geoid height. Also, in addition to this direct practical function, a knowledge of the geoid is of scientific interest in the contribution it makes to the understanding of the Earth's crustal structure. The only feasible method of determining geoid heights over a large area to the required accuracy and density is the gravimetric method.

Since the first gravimetric determination of the geoid of the British Isles in 1979 [6], a number of developments have taken place. The amount of data available, both local and global, has greatly increased, while new methods of computing geoid heights from gravity observations have been devised. The method which was used in the study on which this account is based [1] uses a refinement of Stokes integral. The latter is sometimes characterised as a simple spherical approximation that requires a continuous gravity field and which takes no account of the topography, and on these grounds is considered to be insufficiently accurate for the precise determination that is now required. In fact, given sufficiently dense data coverage, together with the application of recently developed theoretical and practical modifications of the basic integral these objections can be overcome. It is also claimed that the Stokes method requires excessive computation time, but this is now elearly no longer a problem. Thus, with a very large data set and unlimited computing resources, this method becomes a natural choice, and in this 
pure form allowed investigation of the effects of Stokes approximation and assumptions. In this situation the possible advantages of some of the more recently developed methods were not at all obvious, although they could be applicable in other circumstances - with a sparse data set, for instance, or where computing facilities are limited. For example, while the Fast Fourier Transform (FFT) technique is, as its name implies, computationally extremely fast, the transformation to the frequency domain involves a gross simplification of the Stokes kernel. Least squares collocation, while theoretically sound, suffers from the practical disability that it generates a system of equations of order equal to the number of observation points, so that whilst it might be appropriate in regions where measurements are sparsely distributed or heterogeneous, its application in an area containing several hundred thousand observations involves the selection of data points, amounting effectively to throwing away information. Furthermore, the method becomes unstable if the data points are too close together. On balance, therefore, it appears that Stokes integral, corrected for ellipsoidal and topographic effects, with data averaged over small cells, is the most suitable and accurate method of determining the geoid of the British Isles.

\section{METHOD}

The well-known Stokes formula for the height of the geoid above the ellipsoid is

where

$$
N=\frac{R}{4 \pi \gamma} \int_{\nu} S\left(\psi^{\prime}\right) \Delta g d \sigma
$$

$$
\begin{aligned}
N & =\text { geoid-ellipsoid separation } \\
R & =\text { radius of the Earth } \\
\gamma & =\text { mean value of gravity } \\
S(\psi) & =\text { Stokes' function or kernel } \\
\psi & =\text { angular distance to the gravity anomaly } \\
\Delta \mathrm{g} & =\text { free-air gravity anomaly } \\
d \sigma & =\text { element of the unit sphere } \sigma
\end{aligned}
$$

the integration being taken over the unit sphere. The gravity anomaly is the difference between the observed value of gravity, reduced to the surface of the geoid by means of the so-called free-air correction, and normal gravity, the theoretical value of gravity on the surface of the reference ellipsoid.

Stokes function is given by

$$
\begin{aligned}
S(\psi / \gamma)=\operatorname{cosec}(\psi / 2)-6 \sin (\psi / 2)+1-5 \cos (\psi / \gamma) & \\
& -3 \cos (\psi) \ln \left[\sin (\psi / 2)+\sin ^{2}(\psi / 2)\right]
\end{aligned}
$$

An important alternative formulation of Stokes' function in terms of Legendre functions is

$$
S(\psi)=\sum_{n=-2}^{x} \frac{2 n+1}{n-1} P_{n}(\cos \psi)
$$

where $P_{n}(\cos \psi)$ is the Legendre function of degree $n$. This formula enables the 
value of $S(\psi)$ up to any given degree to be determined, and is of direct application in the analysis that follows.

At any given point the geoid height is given by the integration of gravity anomalies, multiplied by the appropriate values of Stokes function, over the entire Earth. However, as the distance from the computation point increases, the degree of accuracy with which the gravity anomalies must be known diminishes. This can be seen from the fact that Stokes function is infinite at the computation point, and has the values 1163 at $\psi=0.1^{\circ}, 125$ at $1^{\circ}$ and from about $26^{\circ}$ oscillates between about \pm 3 . The global distribution of terrestrially surveyed gravity values is patchy, and in many areas non-existent, but in recent years this deficit has been overcome by the creation of global geopotential models, based on the available gravity data, but powerfully reinforced by results from the analysis of the perturbations in the orbital paths of satellites and from data derived from satellite altimetry over the oceans. Such models are capable of representing the long wavelength elements of the global gravity field, and provide an acceptable representation of it for use in conjunction with a dense and accurate local gravity survey. The expression for geoid heights derived from a geopotential model, $N_{k}$, takes the following form

$$
N_{G}=\frac{G M}{r}\left\{\sum_{n=2}^{n \text { matz }} \sum_{n=0}^{n}\left(\frac{a}{r}\right)^{n}\left[\bar{C}_{x m} \cos m \lambda+\bar{S}_{t a n t} \sin m \lambda\right] \bar{P}_{n m i}(\sin \phi)\right\}
$$

where

$$
\begin{aligned}
G= & \text { the Newtonian gravitational constant } \\
M= & \text { mass of the Earth, including the atmosphere } \\
r= & \text { distance from Earth's mass-centre } \\
a= & \text { Earth's equatorial radius } \\
\phi, \lambda= & \text { geocentric latitude and longitude } \\
\bar{C}_{n m}, \bar{S}_{n m}= & \text { fully normalised harmonic coefficients of degree } n \\
& \text { and order } m, \text { reduced for the zonal harmonics of } \\
& \text { the reference ellipsoid } \\
\bar{P}_{n m m}(\sin \phi)= & \text { fully normalised associated Legendre function of } \\
& \text { degree and order } n, m \\
n m a x= & \text { maximum degree of geopotential model. }
\end{aligned}
$$

The simple application of the so-called fundamental equation of physical geodesy together with Bruns' equation [2] enables the sum of the harmonic components of the gravity anomalies up to any given degree to be calculated from the same series as the geoid heights, namely

$$
\Delta g_{n \text { nax }}=\frac{G M}{r^{2}}\left\{\sum_{n=2}^{n \text { nin }} \sum_{n=0}^{n}(n-1)\left(\frac{a}{r}\right)^{n}\left[\bar{C}_{n m} \cos m \lambda+S_{n m} \sin m \lambda\right] \bar{P}_{n m}(\sin \phi)\right\}
$$

where $\Delta g_{n \text { max }}$ is the total component of the gravity anomaly up to degree $n \max$ and the remaining notation is as in equation (5).

If, in the area of interest, the long-wavelength element of the geoid heights is calculated from such a global model, then it is only necessary to add the difference 
due to the local short-wavelength gravity field. In other words, the gravity anomalies to be used in Stokes integral are referred to the surface of the figure represented by the model instead of to the reference ellipsoid, and this is done by subtracting the gravity anomalies of the same degree as the model geoid heights from the observed gravity anomalies. This greatly reduces the effect of the spherical approximation used in the derivation of Stokes' integral.

It would seem that the two quantities in the integrand of Stokes' integral should be of the same degree, so that if the gravity anomalies are reduced by degree $M$, the maximum degree of the geopotential model employed, then so should Stokes function. Subtracting $\Delta g_{M}$, given by equation (6), from $\Delta g$ to obtain the residual gravity anomaly $\Delta g_{3+1}$ (where from now on the subscript $M+1$ denotes the summation of terms of degree greater than $M)$, and $S(\psi)_{M}$, given by equation (4), from $S(\psi)$ to get the corresponding value of Stokes function, equation (2) is replaced by

$$
N=N_{G_{M}}+\frac{R}{4 \pi \gamma} \int_{\sigma} S_{M+1}(\psi) \Delta g_{M+1} d \sigma
$$

where $N_{G_{M}}$ is the geoid height given by the geopotential series to degree and order $M$. However, because of the orthogonality relationship in the integration of harmonic products [2], the following relationships are valid

$$
\int_{\sigma} S_{M+1}(\psi) \Delta g_{M+1} d \sigma=\int_{\pi} S_{M+1}(\xi) \Delta g d \sigma=\int_{\sigma} S(\psi) \Delta g_{M+1} d \sigma
$$

and, as described later, it is the third of these expressions that is actually employed in the computation.

The accuracy of a geopotential model is dependent on that of the observational data from which it is derived, while its horizontal and vertical resolution are expressed by its degree and order. Clearly the more accurate and abundant the data, the higher the degree and order to which the coefficients of the model can confidently' be computed. Thus for any given model it is possible to deduce the distance from the computation points beyond which the model alone can be regarded as representing the long and medium wavelengths of the gravity field. The application of Stokes integral can therefore be limited to within this distance $\psi_{0}$, the so-called integration cap size, the area within this spherical cap $\sigma_{0}$ being covered by an intensive local terrestrial gravity survey. It is evident that this procedure results in a considerable reduction in both data requirements and computational effort, but nevertheless we need to determine the error that is committed by assuming that the geopotential model actually represents the gravity field in the zone exterior to the cap, and to find some means of minimising it. Calling this error $\delta N$, then, making use of the third expression in equation (8)

$$
\delta N=\frac{R}{4 \pi \gamma} \int_{\sigma-\sigma_{0}} S(\psi) \Delta g_{M+1} d \sigma
$$

where $\sigma-\sigma_{0}$ is the region exterior to the cap. By a procedure originally due to Molodensky [5] the Stokes kernel in equation (7) can be modified in such a way as to minimise, according to a least-squares criterion, the effect of neglecting this term, but it has been found that a development of this method, due to Meissl [4], which forces the Stokes kernel to zero at the cap boundary, reduces the truncation 
errors, using an elegant and mathematically simple modification. The Meissl modification of Stokes function is given by

$$
S^{m e}(\psi)=S(\psi)-S\left(\psi_{0}\right) .
$$

The general form of Meissl's and other kernel modifications is in fact considerably more complex than this, but justification for the adoption of this form was provided by a comprehensive analysis [1].

Thus, finally, the equation for the geoid height becomes

$$
N=N_{G_{M}}+\frac{R}{4 \pi \gamma} \int_{\sigma_{\|}} S^{m e}(\psi) \Delta g_{M+1} d \sigma
$$

The practical implementation of Stokes integral requires a number of preliminary steps. Firstly, since the gravity field is not known as a continuous function but as discrete values, the integral must be expressed in a form suitable for numerical integration i.e. as a summation. Also, to facilitate the computation process it is convenient to sort the gravity anomalies into small blocks, with meridians and parallels as sides and approximately square in shape, and to average the anomalies within them in some way. The wavelength and accuracy of the resulting geoid heights are clearly a function of the size of the blocks and the number of observations they contain. The numerical version of equation (11) thus becomes

where

$$
N=N_{C_{M}}+\frac{R}{4 \pi \gamma} \sum_{k} S^{m e}\left(\psi_{m}\right)_{k} \overline{\Delta g_{k}} A_{k}
$$

$S^{m e}\left(\psi_{m}\right)_{k}=$ Stokes function with Meissl's modification at the

centre of the $k$ th block, distance $z_{m}$

$\overline{\Delta g_{k}}=$ residual mean free air anomaly for the block

$A_{k}=$ area of block in angular units.

\section{DATA}

The data available for this study comprised:

1. 156284 land gravity observations covering England, Scotland and Wales.

2. 11256 land gravity observations over Northern Ireland.

3. 20101 land gravity observations over the Republic of Ireland.

4. 64104 marine gravity observations in the surrounding oceans.

5. 74263 gridded marine gravity observations on a $4 \mathrm{~km}$ grid, overlapping to a certain extent the point values above.

6. 130317 spherical harmonic coefficients of the Ohio State University geopotential model OSU91A, to degree and order 360 [8].

7. Two digital elevation models (DEM) were available:

(i) Southern England and Wales $-200 \mathrm{~m}$ resolution.

(ii) Northern England and Scotland $-1 \mathrm{~km}$ resolution.

For Ireland and small areas of Britain not covered by (i) and (ii) a $2^{\prime} \times 4^{\prime}$ DEM was constructed from mean heights of the gravity observations.

The extent of the gravity coverage is shown in Fig. 1. 


\section{Computation AND REsults.}

The gravity data sets above were filtered to detect gross errors and assembled into a single uniform data set with a standard format. The free-air anomalies were obtained by applying a second-degree free-air reduction as given in [2], i.e.

$$
\delta g_{f a}=\frac{2 \gamma_{a}}{a}\left(1+f+m-2 f \sin ^{2} \phi\right) H-\frac{3 \gamma_{a}}{a^{2}} H^{2}
$$

where

$$
\begin{aligned}
f & =\text { flattening of the GRS80 ellipsoid } \\
m & =\omega^{2} a / \gamma_{a}, \text { the centrifugal ratio } \\
\omega & =\text { Earth's angular velocity } \\
a & =\text { Earth's equatorial radius } \\
\gamma_{a} & =\text { normal gravity at the Equator } \\
\phi & =\text { latitude } \\
H & =\text { height above the geoid }
\end{aligned}
$$

and a correction for the mass of the atmosphere. The latter consisted of a fifth degree polynomial fitted to averages of values determined from the U.S. and COSPAR standard atmospheres [3]. This gave

$$
\begin{aligned}
\delta g_{a c}= & 0.871-1.0298 \times 10^{-4} H+5.3105 \times 10^{-9} H^{2} \\
& -2.1642 \times 10^{-13} H^{3}+9.5246 \times 10^{-18} H^{4}-2.2411 \times 10^{-22} H^{5}
\end{aligned}
$$

where $\delta g_{a c}$ is in milligals and $H$ is in metres.

The resulting free-air anomalies were then sorted into compartments of 2 ' extent in latitude and $4^{\prime}$ in longitude, roughly $4 \mathrm{~km}$ square, with up to 20 values per compartment. Various averaging techniques were considered, including the regression method used in [6], averaging the Bouguer anomalies and then restoring the topography using the DEM, a surface fitting and interpolation, and a straight mean of the free-air anomalies. It was considered that there were insufficient observations in each compartment for satisfactory application of the regression method, while tests with the other three produced closely similar results, so the straight averaging method was adopted. A topographic correction, to account for the residual attraction of the masses condensed onto the geoid by the free-air or condensation reduction was applied to these mean free-air anomalies as follows [1]

$$
\delta g_{t}=\frac{G \rho R^{2}}{2} \sum_{k} \frac{\left(H^{\prime}-H\right)^{2}}{l^{3}} A_{k}
$$

where

$$
\begin{aligned}
G & =\text { gravitational constant } \\
\rho & =\text { Earth's crustal density } \\
R & =\text { radius of Earth } \\
H & =\text { mean orthometric height of computation point compartment } \\
\bar{H}^{\prime} & =\text { mean orthometric height of distant compartment } \\
l & =\text { distance from computation point } \\
A_{\ell} & =\text { area of block in angular units. }
\end{aligned}
$$




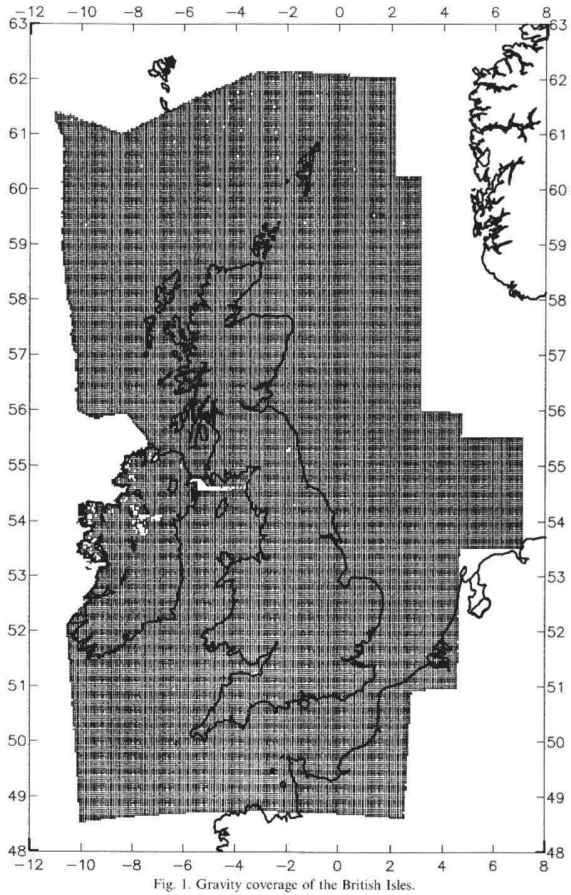

The digital elevation model was used to calculate this correction.

The application of the free-air reduction, whereby the observed gravity is reduced to the surface of the geoid, is equivalent to condensing the topography onto the surface of the geoid (Helmert's condensation reduction, [2]). The effect 
of this transfer of mass is to alter the Earth's potential, so that the surface determined by equation (11) is not exactly the geoid, but a close surface known as the co-geoid, the separation between these two surfaces being known as the indirect effect of the condensation reduction. An expression for this is given in [11], for which the discrete version is

$$
\delta N_{i}=-\frac{\pi G \rho \bar{H}^{2}}{\gamma}-\frac{G \rho R^{2}}{6 \gamma} \sum_{k} \frac{\left(H^{\prime 3}-H^{3}\right)}{l^{3}} A_{i}
$$

where $\gamma$ is normal gravity and the remaining notation is as for equation (15).

The first term of this expression represents the effect of the infinite Bouguer plate, while the second term takes into account the short wavelength variations due to the topography. A digital elevation model is used to determine the correction for the indirect effect, which is applied directly to the final geoid heights. The rapid decay in the value of $l^{-3}$ in the second term allows summation to be limited to a radius of $60 \mathrm{~km}$.

A matter of fundamental importance was the determination of the size of the integration cap. It needs to be as small as possible in order to minimise the computational labour, but accuracy must not be prejudiced on this account, so the value adopted must be based on analytical criteria rather arbitrary selection. Since it depends not only on the degree and order of the geopotential model, but also on the stability of Meissl's modification of the Stokes kernel, this was the subject of an intensive and complex analysis, together with rigorous testing [1]. An examination of the coefficients of the selected geopotential model [8] showed that from degree 257 onwards the standard errors begin to exceed the magnitude of the coefficients themselves and therefore it was decided to truncate the model at this point. Now it is well-known that a geopotential model should be accepted in its entirety and that in general it is not legitimate to isolate terms from it, since this will incur aliasing errors, edge effects, etc. However, while this would be a significant consideration at the lower degrees, at this end of the spectrum degree 257 accounts for $98 \%$ of the total geopotential model height, so that any errors on this account are very small, but in any case are taken into account by the second term of the geoid height evaluation (equation (11)), where the reduced gravity anomaly is the residue of whatever has not been included in the geopotential model. In effect, whatever has been removed from the gravity anomaly is subsequently restored. As a result of this analysis, a optimum cap radius was found to be $1.951^{\circ}$, and this value was confirmed by an independent method.

The geoid heights were evaluated at the centre of each $2^{\prime}$ latitude $\times 4^{\prime}$ longitude compartment in the latitude range $48^{\circ}$ to $63^{\circ}$ North and longitude range $12^{\circ}$ West to $8^{\circ}$ East, a total of 135000 points. Because of the rapid change in Stokes function for small values of $\psi$, the compartment midpoint values are not truly representative of the mean value and require a correction on that account. The correction, derived in [9], takes the form of a factor $f\left(\psi_{m}\right)$ by which the Stokes kernel is multiplied

$$
f\left(\psi_{m}^{\prime}\right)=\frac{\psi_{m}^{\prime}}{\delta \psi} \ln \left(\frac{2 \psi_{m}+\delta \psi}{2 \psi_{m}-\delta \psi}\right)
$$

where $\delta \psi$ is the distance across the compartment and $\psi \psi_{m}$ is the distance to the 
GRAVIMETRIC DFTERMINATION OF THE GEOID

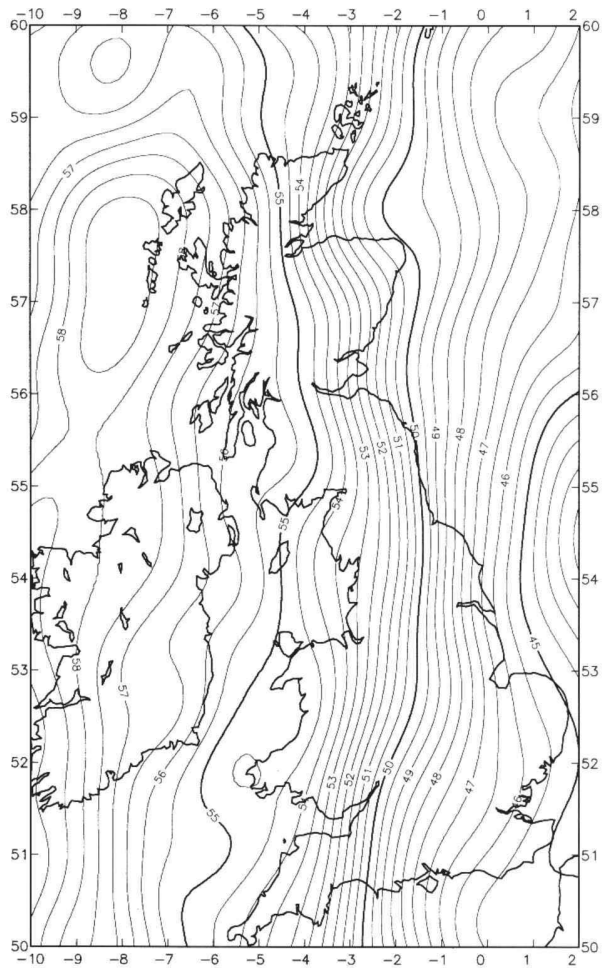

Fig. 2. OSU91A geoid height contours on GRS80 ellipsoid, degree and order 257. Contour interval $0.5 \mathrm{~m}$. 


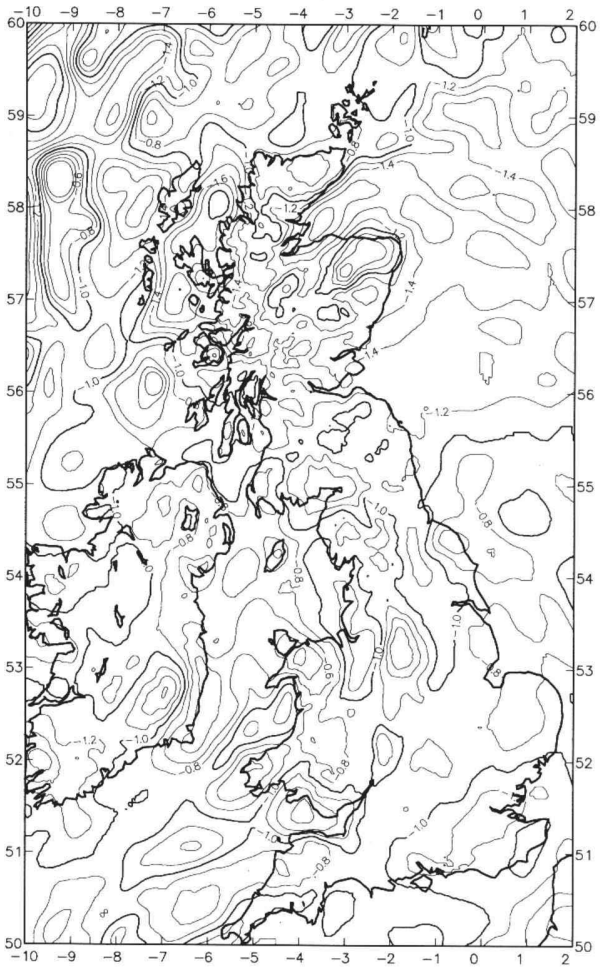

Fig. 3. Residual gcoid contours determined from the Stokes integration-GRS80. Contour interval $02 \mathrm{~m}$. 
GRAVIMETRIC DETERMINATION OF THF GEOID

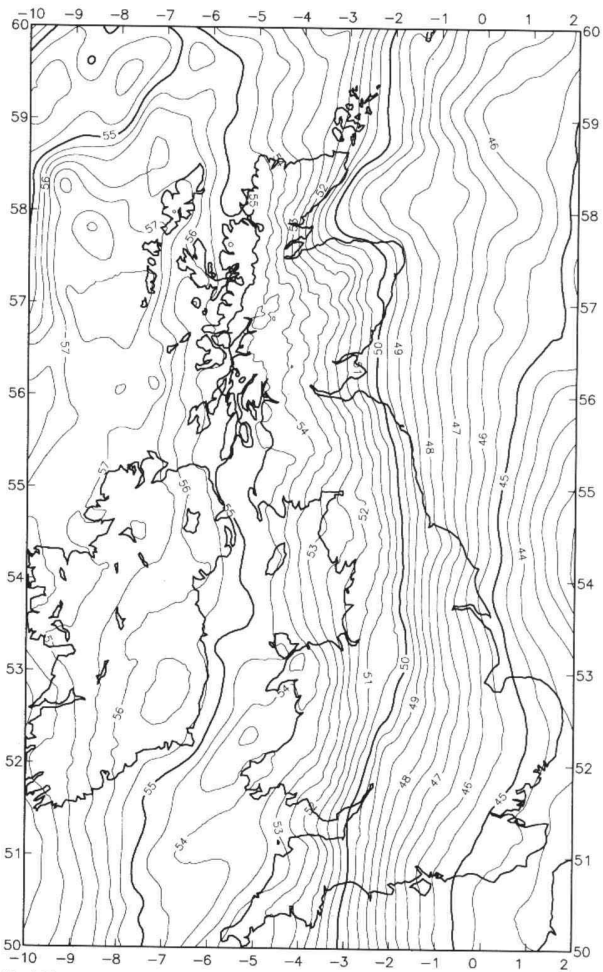

Fig. 4. Final gravimetric geoid height contours, the sum of the components shown in Figs 2 and 3 , GRS80. Contour interval $0.5 \mathrm{~m}$. 
centre of the compartment. For the compartments employed here the factor ranged from 1.0217 to 1.0003 at the cap boundary.

For the compartment centred on the computation point, where the Stokes kernel becomes infinite, a special procedure, as given in [2] and [9] was applied. Denoting the contribution to the geoid height from this innermost compartment by $\delta n$, then

$$
\delta n=\frac{\Delta g_{M 11} R}{\gamma} \sqrt{\frac{A}{\pi}} .
$$

The results from the integration cap, including the innermost zone, were added to the geopotential model heights and indirect effect to produce the final geoid heights.

The stages in the computation are illustrated in Figs 2, 3 and 4, which show, respectively, the OSU91A geoid model contours up to degree and order 257 , the component obtained from the integration of the gravity anomalies, and the final gravimetric geoid height contours, all on GRS80. The Stokes contribution ranges from $0 \cdot 00$ to $-2 \cdot 30$ metres. For comparison, contours for the complete $360 \times 360$ OSU91A model are shown in Fig. 5, and it can be seen that the difference from the 257 degree version in Fig. 2 are barely perceptible.

\section{Conclusions}

Although a rigorous error analysis of the Stokes method is difficult and has never been done, a careful analysis of the possible error sources leads to the conclusion that the standard error of the absolute geoid height determination is of the order of $\pm 8 \mathrm{~cm}$, although there may be a small residual bias, while for geoid height differences over distances of up to $100 \mathrm{~km}$ it is in the region of $1 \mathrm{~cm}$. GPS observations were available at 54 primary triangulation pillars but unfortunately the orthometric height determination at these points was mainly by tertiary levelling, while most of the GPS observations were made by single frequency instruments. Nevertheless 46 of the results lay within $\pm 20 \mathrm{~cm}$ of the gravimetric value, evenly balanced about zero. The contours of the GPS geoid heights are shown in Fig. 6, and considering the small number of points involved, the agreement with the gravimetric values is remarkably good. Because of the north-south orientation of the contours, what does not show up is the tendency for a progressive northward negative increase in the differences GPS minus gravimetric, amounting to $-53 \mathrm{~cm}$ at the northernmost point. While the comparisons are not sufficiently numerous to provide statistically significant confirmation, this tends to corroborate the findings in [10] that there is a northward rise in sea-level, and hence the geoid, relative to the levelling, of $5.3 \mathrm{~cm}$ per degree of latitude.

Thus we may conclude that the new gravimetric geoid of the British Isles has provided an advance over the first determination through the use of more data and refined computational techniques to yield a higher resolution and accuracy, with applications to GPS positioning and levelling, and to geophysics. 


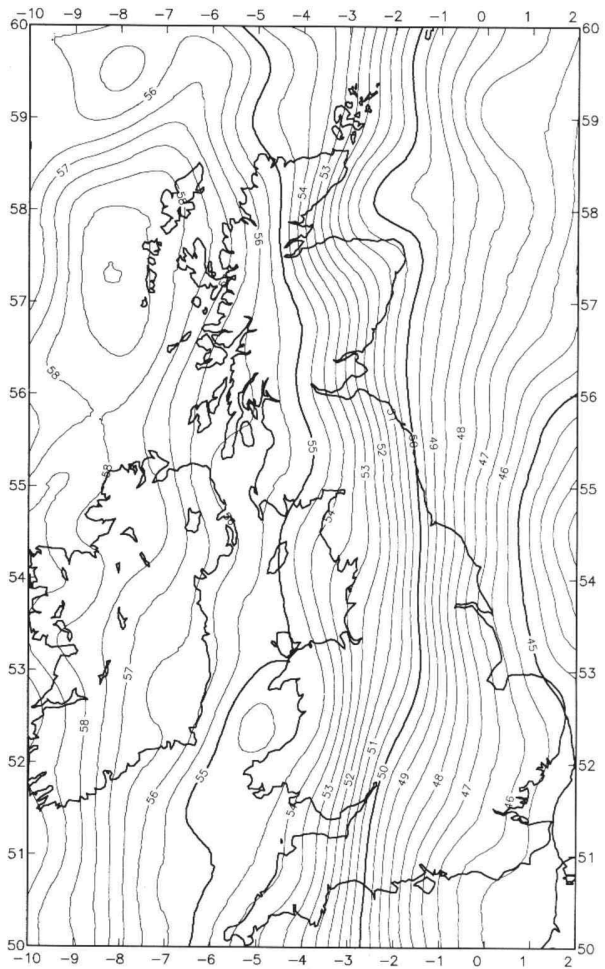

Fig. 5. OSL91A geoid height contours on GRS80 ellipsoid, degree and order 360. Contour interval $0.5 \mathrm{~m}$. 
W. E. FEATHERSTONE AND J. G. OLLIVER

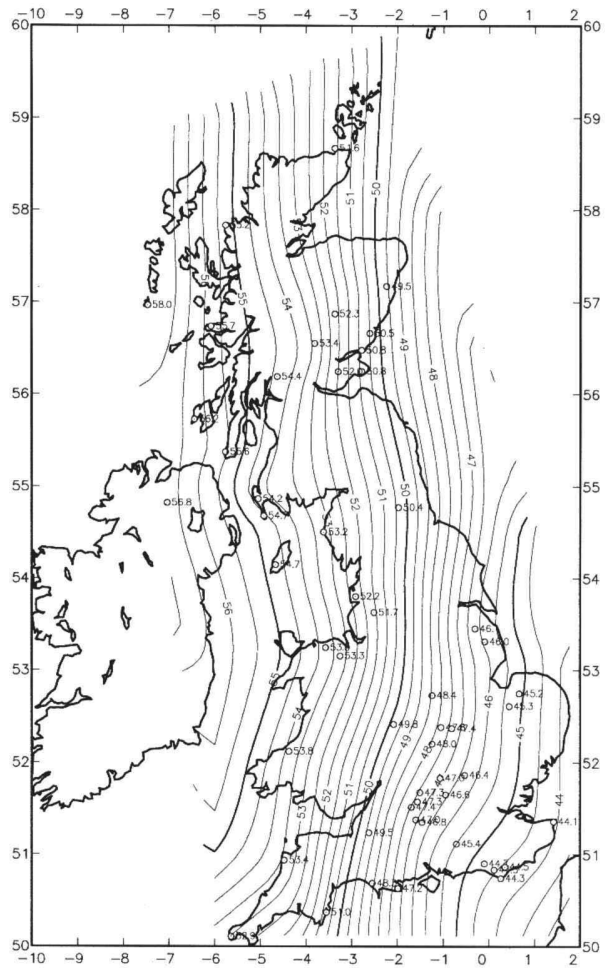

Fig. 6. Geoid contours on GRS80 ellipsoid from GPS observations at 54 Principal Retriangulation (Ordnance Survey Scientific Network 1980) points. Contour interval $0.5 \mathrm{~m}$. 


\section{ACKNOWLEDGEMENTS}

We are greatly indebted to the individuals and organisations that supplied the data for this study. These include British Geological Survey, Ordnance Survey of Great Britain, Geological Survey of Northern Ireland, Professor R. H. Rapp, Ohio State University, Professor T. Murphy, Dublin Institute of Advanced Studies, Mr Strang van Hees, Delft University and Dr Philippa Berry, de Montfort University. The work was supported by a grant from the Natural Environment Research Council.

\section{References}

1. Featherstone, W. E., 1992. A GPS-controlled granimetric determination of the geoid of the British Isles. D.Phil thesis, Oxford University.

2. Heiskanen, W. A. \& Moritz, H., 1967. Physical geodesy. W. H. Freeman and Co., San Francisco and London.

3. International Association of Geodesy, 1971. Geodetic reference system 1967. Burcau Central de l'Association Internationale de Géodésie, Paris.

4. Meissl, P., Preparations for the numerical etahation of second-order Molodensky-type formulas. Report No. 163, Department of Geodetic Science and Surveying. Ohio State University.

5. Molodenskii. M. S., Eremeev, V. F. \& Yurkin, M. I, 1960. Methods for study of the external gratitational field and figure of the earth. Israel Program for Scientific Translations, Jerusalem 1962.

6. Olliver, J. G., 1980. The gravimetric geoid of Great Britain and Ireland, Geophysical Journal of the Royal Astronomical Society, 63: 253-270.

7. Olliver, J. G., 1992. Space-derived geoid maps of Great Britain. Survey Review, 31: 310-320.

8. Rapp. R. H., Wang. Y. M. \& Pavlis, N. K., 1991. The Ohio State 1991 geopotential and sea surface topography harmonic coefficient models. Report No. 410. Department of Geodetic Science and Surveying, Ohio State University.

9. Strang van Hees, G. L., 1990. Stokes' formula using fast Fourier techniques. Mamuseripta Geodaetica, 15: 235-239.

10. Thompson, K., 1980. An analysis of British monthly mean sea level. Geophysical Journal of the Royul Astronomical Society, $63: 57-73$.

11. Vaniček, P., Kleusberg. A., Chang, R., Fashir, H., Christou, N., Hofman, M., Kling, T. \& Arsenault, T., 1986. The Canadian geoid, Geodetic Survey of Canada, Contract Report 86-001. 